\title{
Nonlinear Cointegration and Nonlinear Error Correction: Record Counting Cointegration Tests
}

\author{
ALVARO ESCRIBANO $^{1}$, ANA E. SIPOLS ${ }^{2}$, AND $^{2}$ \\ FELIPE APARICIO 3
}

${ }^{1}$ Department of Economics, Universidad Carlos III de Madrid, Spain

${ }^{2}$ Department of Statistics, Universidad Rey Juan Carlos, Madrid, Spain

${ }^{3}$ Department Statistics, Universidad Carlos III de Madrid, Spain

\begin{abstract}
In this article we propose a record counting cointegration (RCC) test that is robust to nonlinearities and certain types of structural breaks. The RCC test is based on the synchronicity property of the jumps (new records) of cointegrated series, counting the number of jumps that simultaneously occur in both series. We obtain the rate of convergence of the RCC statistics under the null and alternative hypothesis. Since the asymptotic distribution of RCC under the null hypothesis of a unit root depends on the short-run dependence of the cointegrated series, we propose a small sample correction and show by Monte Carlo simulation techniques their excellent small sample behaviour. Finally, we apply our new cointegration test statistic to several financial and macroeconomic time series that have certain structural breaks and nonlinearities.
\end{abstract}

Keywords Cointegration; Counting statistics; Jumps; Nonlinearity; Ranges; Robustness; Small sample corrections; Structural breaks; Unit roots tests.

Mathematics Subject Classification 37M10; 62M10.

\section{Introduction}

Granger (1981) introduced the concept of cointegration and with the contribution of Engle and Granger (1987) and Johansen (1991) this concept has achieved immense popularity among econometricians and applied economists. Only a few recent papers have been dedicated to the simultaneous consideration of nonstationarity and nonlinearity, even though many people agree that those are likely characteristics of many macroeconomic and financial economic relationships. Granger (1995) discussed the concepts of long-range dependence in mean and extended memory that generalize the linear concept of integration, $I(1)$, to a nonlinear framework. 
On the other hand, there are interesting empirical macroeconomic applications where nonlinearity has been found in a nonstationary context and therefore, there is a need to justify those results econometrically.

Most unit root tests, like those of Dickey and Fuller (1979) or Phillips and Perron (1988), are not robust to outliers, Franses and Haldrup (1994); nor to structural breaks, Perron (1990); nor to nonlinear transformations, Granger and Hallman (1991) and Aparicio et al. (2006a). Therefore, tests for noncointegration based on the augmented Dickey and Fuller (ADF) test applied to the residuals of the cointegrating relationship, Engle and Granger (1987), have size distortions and losses in power. Aparicio et al. (2004, 2006a) analyzed the asymptotic properties of a new range unit root (RUR) test and provide evidence of their nice behavior by Monte Carlo simulation of nonlinearities and structural breaks and by some empirical applications.

In this article we analyzed the properties of a RCC test that is robust to monotonic nonlinear transformations and structural changes. This testing procedure works with the ranges, instead of using the actual variables. The range for a given time $t$ is defined as the difference between the cumulative maximum and the cumulative minimum at that time $t$. The first differences of the ranges are called the jumps and they represent the arrival of a new maximum or minimum (new record). The RCC test analyzed in this article is based on counting the synchronicity of the jumps (new maximum or minimum) of the cointegrated series. This statistic counts the number of jumps that simultaneously occur in both series. We compare the properties of the well-known non cointegration test (see Engle and Granger [EG], 1987), with those of RCC. We show by Monte Carlo simulations how EG dramatically fails in the presence of nonlinearities and structural breaks. These wellknown results are general and affect most of the available tests of cointegration or non-cointegration, like Johansen (1991), etc.

We have considered as empirical applications the prices of gold and silver, analyzed by Escribano and Granger (1998) and the UK money demand from 1878 to 2000, analyzed by Escribano (2004). Those data sets were selected because there was evidence that the series were cointegrated only after explicit treatments of nonlinearities or structural breaks or both. In this article, however, we find evidence of cointegration without any previous treatment (prefiltering) of those problems.

\section{Analysis Based on Record Counting Cointegration (RCC)}

In this section we introduce nonparametric methods to analyze cointegration that do not impose restrictions on the functional form relating the variables. Some of those procedures are robust to certain types of structural breaks (or level shifts) and monotonic nonlinear transformations.

Aparicio (1995), Aparicio and Granger (1995), and Aparicio et al. (2006b) propose an alternative nonparametric methodology to test for unit roots. The basic idea behind this approach is to count recursively the number of new records (maximum or minimum) in a time series. We expect nonstationary cointegrated series to have many more new corecords (or synchronous new records) than noncointegrated series or stationary series. To be more precise we need to introduce some concepts and definitions that are useful in the rest of the article.

Definition 1. Given a time series $x_{t}$ we define the sequence of extremes of $x_{t}$ as the sequence of $x_{1, t}=\min \left\{x_{1}, \ldots, x_{t}\right\}$ and $x_{t, t}=\max \left\{x_{1}, \ldots, x_{t}\right\}$, when $t=1,2, \ldots, n$. 
Definition 2. Let $n$ be the sample size, the sequence of ranges of $x_{t}$ is defined as

$$
R_{t}^{(x)}=x_{t, t}-x_{1, t} \text { for } t=1, \ldots, n .
$$

Definition 3. Given the sequence of ranges we can define the sequence of jumps (new records) as the sequence of first differences of ranges:

$$
\Delta R_{t}^{(x)}=R_{t}^{(x)}-R_{t-1}^{(x)} .
$$

The series of new records $\Delta R_{t}^{(x)}$ is positive or zero. When there is a new record (maximum or minimum) in $\left\{x_{1}, \ldots, x_{t}\right\}$, the first difference of the ranges will be positive at time $t, \Delta R_{t}^{(x)}>0$. A statistics record was proposed by Aparicio et al. (2006a) for robust unit root testing. When the original series is stationary, with finite variance, the series of first differences of the ranges are positive at the beginning of the sample and the rest is almost a sequence of zeros. On the contrary when the series are nonstationary $I(1)$ new records appear with positive probability as the sample increases. The key idea relied on the different vanishing rates of the longrun frequency of a new record, $n^{-1} \sum_{t=1}^{n} \mathbf{1}\left(\Delta R_{t}^{(x)}>0\right)$, for an $I(1)$ and an $I(0)$ time series, in such a way that the normalized long-run frequency of records

$$
J_{x}^{(n)}=n^{-1 / 2} \sum_{t=1}^{n} \mathbf{1}\left(\Delta R_{t}^{(x)}>0\right)
$$

converged in probability to zero under the alternative of stationary, and to a nondegenerate positive random variable under the null hypothesis of a unit root. A well-known result from extremal theory is that the statistical properties of records from iid sequences of random variables are shared by a wide class of dependent stationary time series (see for instance, Leadbetter and Rootzén, 1988; Lindgren and Rootzén, 1988). This prompts the question of whether short-run dependencies and cross-dependencies may have an impact on a record-based test for cointegration. Then, one important alternative model to consider in this context is the case of dependent random walks.

For plots of the sequence of ranges $R_{t}^{(y)}$ and $R_{t}^{(x)}$ of Models 1 to 4 below, see Aparicio et al. (2006b). The following models are useful to motivate the new approach we are proposing with parameter values equal to $\alpha=0.5, b=0.6$, where $e_{t, 0}, e_{t, 1}, e_{t, 2}$ are standard normal distributions and are mutually independent, $\operatorname{Nid}(0,1)$.

Model 1 (Linear cointegration): $x_{t}=w_{t}+e_{t, 1}, y_{t}=\alpha w_{t}+e_{t, 2}$ where $w_{t}=w_{t-1}+e_{t, 0}$. Model 2 (Nonlinear cointegration): $\quad x_{t}=w_{t}+e_{t, 1}, y_{t}=\alpha w_{t}^{2}+e_{t, 2}$ where $w_{t}$ $=w_{t-1}+e_{t, 0}$.

Model 3 (Independent random walks): $x_{t}=x_{t-1}+e_{t, 1}, y_{t}=y_{t-1}+e_{t, 2}$.

Model 4 (Random walks with short-run dependence $(a \neq 0)$ ): $\Delta x_{t}=e_{t, 1}, \Delta y_{t}=$ $a \Delta x_{t}+e_{t, 2}$.

Figure 1 shows the cross plots of the sequences of new records or first difference of ranges for the different models. From the cross plot of Fig. 1 it is clear that dependent but not cointegrated random walks (d) have several new records synchronized indicating some small number of corecords. This property will likely reduce the power of the RCC test if the short-run correlation is high relative to the cointegration relationship. The idea that cointegrated series imply arrivals of highly 


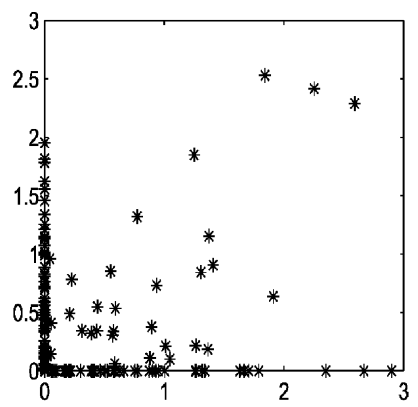

(a)

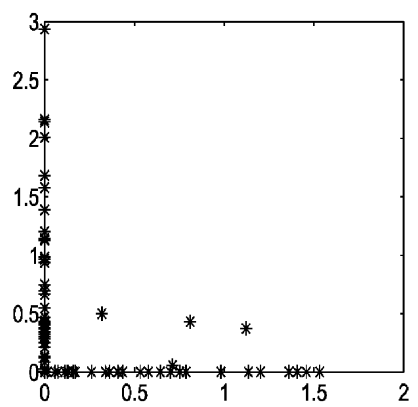

(c)

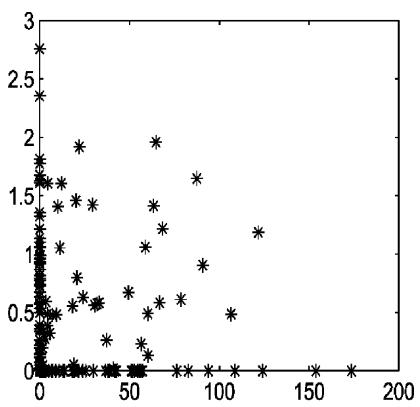

(b)

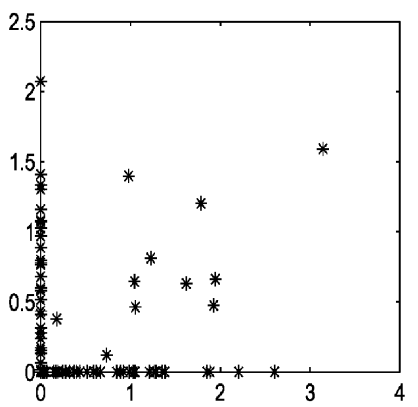

(d)

Figure 1. Cross plots of $\Delta R_{t}^{(y)}$ and $\Delta R_{t}^{(x)}$ for pairs of series that are (a) linearly cointegrated (model 1), (b) nonlinearly cointegrated (model 2), (c) independent random walks (model 3), and (d) random walks with short-run dependence (model 4).

synchronized new records suggests the following nonparametric test statistic that we called RCC

$$
\mathrm{RCC}_{x, y}^{(n)}=\frac{\sum_{t=1}^{n} \mathbf{1}\left(\Delta R_{t}^{(x)}>0\right) \mathbf{1}\left(\Delta R_{t}^{(y)}>0\right)}{\log (n)},
$$

where the product of the two indicator functions $\mathbf{1}\left(\Delta R_{t}^{(x)}>0\right) \mathbf{1}\left(\Delta R_{t}^{(y)}>0\right)$ counts the number of arrivals of new records that are coincident or synchronized (corecords). That is, the RCC counts how many of the total arrivals of the jumps coincide, relative to the $\log (n)$. Therefore, the convergence rates of the test statistic $\mathrm{RC}$ is $\log (n)$.

Theorem 1. Let the processes $x_{t}=\sum_{i=1}^{t} e_{i, 1}$ and $y_{t}=\sum_{i=1}^{t} e_{i, 2}$ for $t=1,2, \ldots, \infty$, where $e_{i, 1}$ and $e_{i, 2}$ are independent continuous zero-mean iid sequences with finite variances and symmetric pdf. Let $R C C_{x, y}^{(n)}$ be the number of joint records of $x_{t}$ and $y_{t}$ in a sample of size $n$ (4). Then

$$
\begin{gathered}
\log (n)^{-1} \mathrm{RCC}_{x, y}^{(n)} \rightarrow 1, \\
E\left\{\mathrm{RCC}_{x, y}^{(n)}\right\}=O(\log n), \\
\operatorname{Var}\left\{\mathrm{RCC}_{x, y}^{(n)}\right\}=O(\log n) .
\end{gathered}
$$


Proof. See the Appendix.

\subsection{Consistency}

If $x_{t}$ and $y_{t}$ are cointegrated, then for some $a \neq 0$ there exists an $I(0)$ sequence $\eta_{t}$ such that $y_{t}=a x_{t}+e_{t, 2}$. Since for large $t, x_{t}$ will dominate $e_{t, 2}$, we can write

$$
\operatorname{RCC}_{x, y}^{(n)}=\frac{\sum_{t=1}^{n} \mathbf{1}\left(\Delta R_{t}^{(x)}>0\right) \mathbf{1}\left(\Delta R_{t}^{(y)}>0\right)}{\log (n)} \simeq \frac{\sum_{t=1}^{n} \mathbf{1}\left(\Delta R_{t}^{(x)}>0\right)}{\log (n)} .
$$

But we know from Aparicio et al. (2006a) that $\sum_{t=1}^{n} \mathbf{1}\left(\Delta R_{t}^{(x)}>0\right)=O\left(n^{1 / 2}\right)$. Thus under the alternative hypothesis of cointegration, the test statistic will satisfy

$$
(\log n)^{-1} \operatorname{RCC}_{x, y}^{(n)} \rightarrow \infty .
$$

\subsection{Invariance Against Monotonic Nonlinearities}

Monotonic transformations preserve the ordering of the observations in any time series, and thus the inter-record times. As a consequence, if we let $f(\cdot)$ and $g(\cdot)$ be a monotonic nonlinear transformations, we must have

$$
\operatorname{RCC}_{f(x), g(y)}^{(n)}=\operatorname{RCC}_{x, y}^{(n)} .
$$

More generally, let $x_{t}$ and $y_{t}$ be $I(1)$ time series variables, and let $x_{t}^{\prime}=f\left(y_{t}\right)+\varepsilon_{t}$, $y_{t}^{\prime}=g\left(x_{t}\right)+\eta_{t}$, where $\left\{\varepsilon_{t}\right\}_{t \geq 1},\left\{\eta_{t}\right\}_{t \geq 1}$ are independent iid sequences with zero mean and finite variances. Since for large values of $t$ the nonlinear transformations $f\left(y_{t}\right) \mathrm{y} g\left(x_{t}\right)$ dominate $\varepsilon_{t}$ and $\eta_{t}$, respectively, the records of $x_{t}^{\prime}\left(y_{t}^{\prime}\right)$ will occur at the same time as $x_{t}\left(y_{t}\right)$. As a consequence, the count of new records before and after the transformation should be the same. That is,

$$
\begin{aligned}
\operatorname{RCC}_{\left(x^{\prime}, y^{\prime}\right)}^{(n)} & =\sum_{t=1}^{n} \mathbf{1}\left(\Delta R_{t}^{\left(x^{\prime}\right)}>0\right) \mathbf{1}\left(\Delta R_{t}^{\left(y^{\prime}\right)}>0\right) \\
& \simeq \operatorname{RCC}_{(x, y)}=\sum_{t=1}^{n} \mathbf{1}\left(\Delta R_{t}^{(x)}>0\right) \mathbf{1}\left(\Delta R_{t}^{(y)}>0\right) .
\end{aligned}
$$

In finite samples, the actual size will oscillate around the nominal size depending on the type of transformations. For example, certain kinds of transformations can emphasize the $I(1)$ part over the $I(0)$ part. This feature may lead, in finite samples, to size fluctuations around the nominal one.

\subsection{Small Sample Performance of the RCC Test: Monte Carlo Simulations}

Let the data generation process (DGP) be the following noncointegrated random walks but with short-run dependence $(a \neq 0), H_{0}: \Delta x_{t}=e_{t, 1}, \Delta y_{t}=a \Delta x_{t}+e_{t, 2}$.

In Table 1 we estimate the quintals of the empirical distribution of the RCC test statistic, under $H_{0}$, for different parameter values of the short-run dependence $(a=0,0.5,1,1.5)$, different sample sizes $n=100,250,500,1000$, and different significance values $v$. The estimated quintals, based on 10,000 Monte Carlo simulations, are shown in Table 1. We observe that for most sample sizes $(n)$ 
Table 1

Quintals of the empirical distribution of RCC for dependent not cointegrated random walks, for different values of parameter $a$ and for different sample sizes $n$

\begin{tabular}{|c|c|c|c|c|c|c|c|c|}
\hline \multirow[b]{2}{*}{$v / n$} & \multicolumn{4}{|c|}{$a=0$} & \multicolumn{4}{|c|}{$a=1$} \\
\hline & 100 & 250 & 500 & 1000 & 100 & 250 & 500 & 1000 \\
\hline 0.01 & 0.21 & 0.18 & 0.16 & 0.18 & 0.43 & 0.54 & 0.64 & 0.71 \\
\hline 0.025 & 0.21 & 0.18 & 0.32 & 0.33 & 0.65 & 0.72 & 0.96 & 1.12 \\
\hline 0.05 & 0.43 & 0.36 & 0.32 & 0.34 & 0.86 & 0.90 & 1.12 & 1.31 \\
\hline 0.10 & 0.53 & 0.54 & 0.48 & 0.52 & 1.86 & 2.08 & 2.28 & 2.42 \\
\hline 0.90 & 2.30 & 2.08 & 2.28 & 2.31 & 3.17 & 3.53 & 4.05 & 4.51 \\
\hline 0.95 & 3.11 & 3.16 & 3.11 & 3.12 & 3.38 & 3.89 & 4.37 & 4.71 \\
\hline
\end{tabular}

the quintals increase with the short-run dependence $(a)$ in small sample sizes and therefore the empirical distribution of RCC is shifted to the right. These simulation results call for the need of a small sample correction of the RCC statistic to make it useful, since in empirical application we do not know the true value of the parameter $a$. One possibility is to prefilter the series (use of instrumental variables, etc.), but this could be complicated if the dependence is nonlinear. A better alternative is to divide the RCC by the number of new records that are synchronized between the first differences of the series. We call this nonparametric statistic the RCC for corrected dependence:

$$
\operatorname{RCC}_{\mathrm{CD}}=\frac{\sum_{t=1}^{n} \mathbf{1}\left(\Delta R_{t}^{(x)}>0\right) \mathbf{1}\left(\Delta R_{t}^{(y)}>0\right)}{\log (n) \sum_{t=1}^{n} \mathbf{1}\left(\Delta R_{t}^{(\Delta x)}>0\right) \mathbf{1}\left(\Delta R_{t}^{(\Delta y)}>0\right)} .
$$

In Table 2 we estimate the quintals of the empirical distribution of the $\mathrm{RCC}_{\mathrm{CD}}$ test statistic, under $H_{0}$, for different parameter values of the short-run dependence $(a=0,1)$, different sample sizes $n=100,250,500,1000$, and different significance values $v$ based on 10,000 Monte Carlo simulations. The asymptotic distribution of the RCC is a good approximation of the distribution of the RCC in moderate

Table 2

Quintals of the empirical distribution of $\mathrm{RCC}_{\mathrm{CD}}$ for dependent not cointegrated random walks, for different values of $a$ and for different sample sizes $n$

\begin{tabular}{|c|c|c|c|c|c|c|c|c|}
\hline \multirow{2}{*}{$\begin{array}{l}\mathrm{RCC}_{\mathrm{CD}} \\
v / n\end{array}$} & \multicolumn{4}{|c|}{$a=0$} & \multicolumn{4}{|c|}{$a=1$} \\
\hline & 100 & 250 & 500 & 1000 & 100 & 250 & 500 & 1000 \\
\hline 0.01 & 0.21 & 0.231 & 0.23 & 0.24 & 0.22 & 0.24 & 0.242 & 0.24 \\
\hline 0.025 & 0.21 & 0.232 & 0.24 & 0.25 & 0.23 & 0.232 & 0.25 & 0.26 \\
\hline 0.05 & 0.45 & 0.38 & 0.35 & 0.378 & 0.41 & 0.33 & 0.332 & 0.376 \\
\hline 0.10 & 0.56 & 0.58 & 0.59 & 0.60 & 0.53 & 0.58 & 0.59 & 0.60 \\
\hline 0.90 & 2.3 & 2.24 & 2.28 & 2.3 & 2.4 & 2.51 & 2.49 & 2.52 \\
\hline 0.95 & 3.32 & 3.27 & 3.42 & 3.42 & 3.53 & 3.54 & 3.57 & 3.6 \\
\hline
\end{tabular}




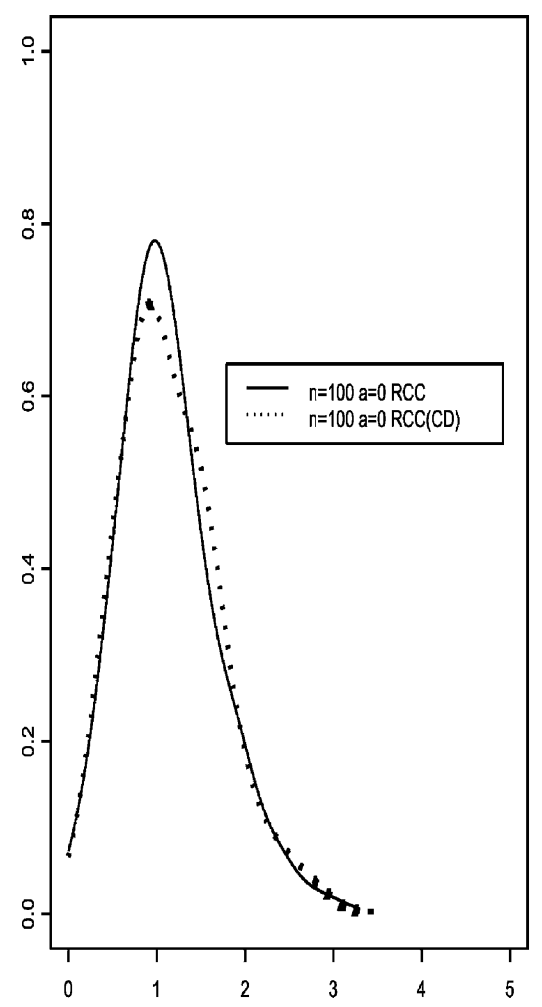

(a)

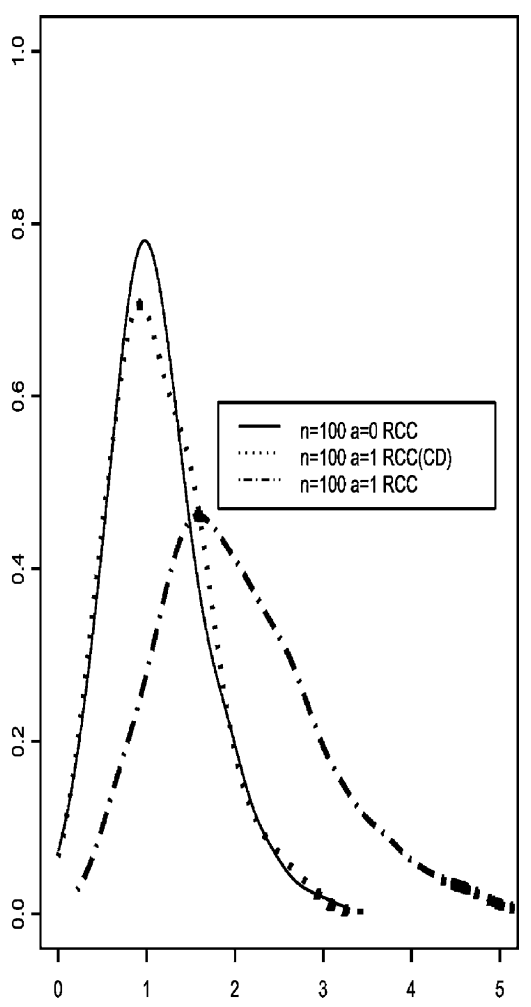

(b)

Figure 2. Nonparametric kernel estimates of the density function of the $\mathrm{RCC}_{\mathrm{CD}}$ and $\mathrm{RCC}$ test statistics under the null hypothesis of no cointegration for $n=100$ and for $a=0,1$.

sample sizes. We can observe the invariance that was obtained with the small sample correction of the RCC statistic for different values of $a$ and different sample sizes $n$. Figures 2 and 3 show the nonparametric estimates of the density function of RCC and $\mathrm{RCC}_{\mathrm{CD}}$ test statistics under the null hypothesis of independent random walks $(a=0)$, with $\operatorname{Nid}(0,1)$ errors and $a=1 ; 1,000$ replications were done using the Epanechnikov kernel.

\section{Size Adjusted Power of the RCC Tests: Monte Carlo Evidence}

We will analyze the power of the RCC tests using the 5\% right tail critical value. The DGP under the alternative hypothesis of cointegration is generated by a bivariate vector error correction model with weakly exogenous variables for the cointegrating parameter vector.

Consider the following restricted VAR model, for the $\left(y_{t}, x_{t}\right)$ vector that is generated by

$$
\left[\begin{array}{ll}
1 & a \\
0 & 1
\end{array}\right]\left[\begin{array}{l}
\Delta y_{t} \\
\Delta x_{t}
\end{array}\right]=\left[\begin{array}{l}
c \\
0
\end{array}\right]+\left[\begin{array}{l}
b \\
0
\end{array}\right](1,-\alpha)\left[\begin{array}{l}
y_{t-1} \\
x_{t-1}
\end{array}\right]+\left[\begin{array}{l}
w_{1 t} \\
w_{2 t}
\end{array}\right],
$$




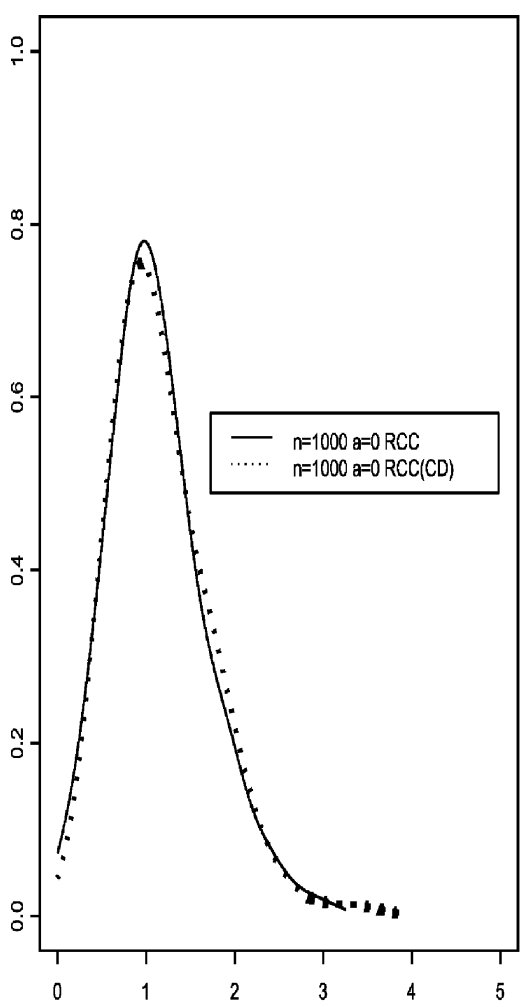

(a)

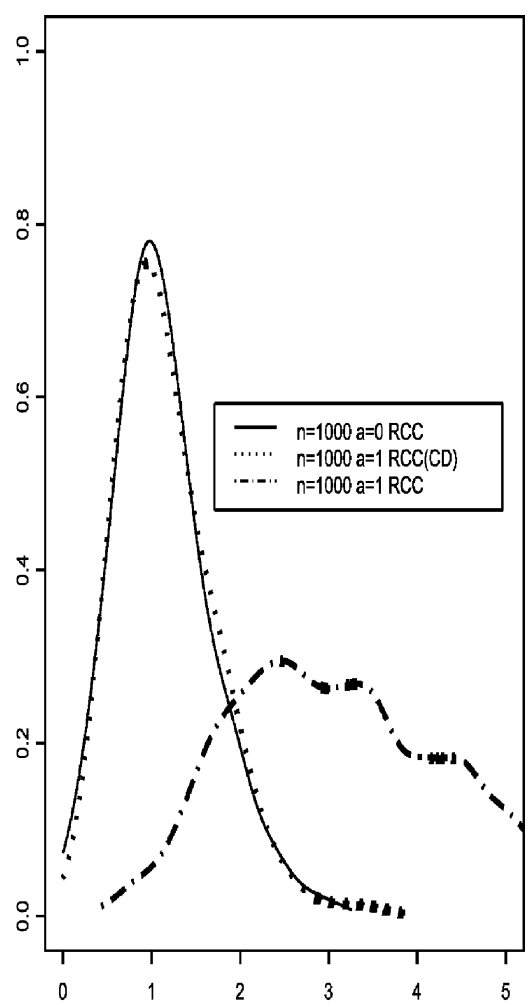

(b)

Figure 3. Nonparametric kernel estimates of the density function of the $\mathrm{RCC}_{\mathrm{CD}}$ and $\mathrm{RCC}$ test statistics under the null hypothesis of no cointegration, for $n=1,000$ and for $a=0,1$.

where

$$
w_{t} \sim \operatorname{Nid}\left(\left[\begin{array}{l}
0 \\
0
\end{array}\right],\left[\begin{array}{ll}
1 & 0 \\
0 & 1
\end{array}\right]\right) .
$$

\subsection{Linear Cointegration}

$H_{1}$ : The alternative hypothesis is a standard linear error correction model (ECM) DGP: $\Delta y_{t}=c+a \Delta x_{t}+b\left(y_{t-1}-\alpha x_{t-1}\right)+w_{1 t}, \Delta x_{t}=w_{2 t}$,

where $\alpha=1$ and $c=0$. We will study the power of different noncointegration tests: the RCC, and the Engle-Granger (EG, 1987), for different parameter values of the parameter $(b)$, say $b=0$ (noncointegration), $b=-0.01,-0.1,-0.25,-0.75$ (cointegration) and different short-run dependence given by $a=0,1$. This DGP follows the parameterization used by Kremers et al. (1992) and Arranz and Escribano (2001). The results of EG are in parenthesis in Table 3.

Since the EG test is based on estimating the true error correction model (DGP), the EG test for noncointegration is more powerful than the RCC. However, the $\mathrm{RCC}$ is more powerful for small values of $b$, slow error correction EC adjustment 
Table 3

Power of the RCC and EG tests for different parameter values of $b, a$, and $n$

\begin{tabular}{lcccccc}
\hline$a \backslash n$ & 100 & 250 & 500 & 100 & 250 & 500 \\
\hline$b(E G)$ & & -0.01 & & -0.1 & \\
0 & $0.4(0.047)$ & $0.4(0.0920)$ & $0.5(0.1590)$ & $0.5(0.38)$ & $0.6(0.99)$ & $0.7(1)$ \\
1 & $0.32(0.09)$ & $0.5(0.12)$ & $0.51(0.21)$ & $0.44(0.49)$ & $0.7(0.99)$ & $0.84(1)$ \\
$a \backslash b$ & & -0.25 & & & -0.75 & \\
0 & $0.6(0.99)$ & $0.8(1)$ & $0.81(1)$ & $0.7(1)$ & $0.9(1)$ & $0.91(1)$ \\
1 & $0.54(0.99)$ & $0.7(1)$ & $0.75(1)$ & $0.6(1)$ & $0.6(1)$ & $0.7(1)$ \\
\hline
\end{tabular}

(see for example $a=0, b=-0.01$ in Table 3 ). Notice that the power of the RCC decreases the larger is $a$.

\subsection{Nonlinear Cointegration and Nonlinear Error Correction}

Escribano (1986, 2004) analyzes ECM models in nonlinear contexts where the cointegration relationship can be linear or nonlinear and the equilibrium correction term also can be linear or nonlinear. He proposes alternative representation theorems for nonlinear error correction (NEC) models based on the concepts of $I(1)$ and $I(0)$ introduced by Escribano $(1986,1987)$ and Escribano and Mira (2002). Escribano (1986, 2004) proposes a methodology to implement parametric and nonparametric ECMS. Using the databases of Friedman and Schwartz (1982) and Ericsson et al. (1998), extended until the year 2000, he implemented this methodology to estimate a nonlinear money demand for the United Kingdom from 1878 to 2000. Within the class of parametric models he discusses cubic polynomial (and rational polynomial) ECMs; see also Hendry and Ericsson (1991) and Ericsson et al. (1998). Nonlinearities can eliminate most of the power of the usual noncointegration test as will be seen in the following Monte Carlo simulations.

\subsection{Power of RCC Against a Nonlinear Cointegrating Relationship}

Consider now that the DGP under the alternative hypothesis is given by the following linear ECM with a nonlinear cointegration relationship.

$H_{1}$ : The alternative hypothesis is a linear ECM but with nonlinear cointegration DGP: $\Delta y_{t}=c+a \Delta x_{t}+b\left(y_{t-1}-g\left(x_{t-1}, \alpha\right)\right)+w_{1 t}, \Delta x_{t}=w_{2 t}$,

where $w_{1 t}$ and $w_{2 t}$ are $\operatorname{Nid}(0,1)$ and are mutually independent errors with $\alpha=1$.

We analyze now the power of the RCC test for different values of $a$ and we compare the results with the EG test (results in Table 4 are in parenthesis). Let the nonlinear cointegration relationship be given by the following polynomial cointegration term $g\left(z_{t-1}, \alpha\right)=z_{t-1}^{j}$. Based on 10,000 replications of the Monte Carlo experiment we compute the empirical power of the tests for parameter values $a=$ 0,1 and values of $b=-0.01,-0.1,-0.25,-0.75$.

As we can see from Table 5, when the ECM is linear but with a polynomial cointegration function RCC test is much more powerful than the EG in all the cases. In particular, we observed that the highest power is obtained for 


\section{Table 4}

Size adjusted power of the RCC test and the EG test (in parenthesis) at 5\% significance level based on $H_{1}$ with $g=z_{t-1}^{j}, j=2,3$, and 4, for different sample sizes $n$, different values of parameter $b$, and fixing the parameter $a$ at $a=0$

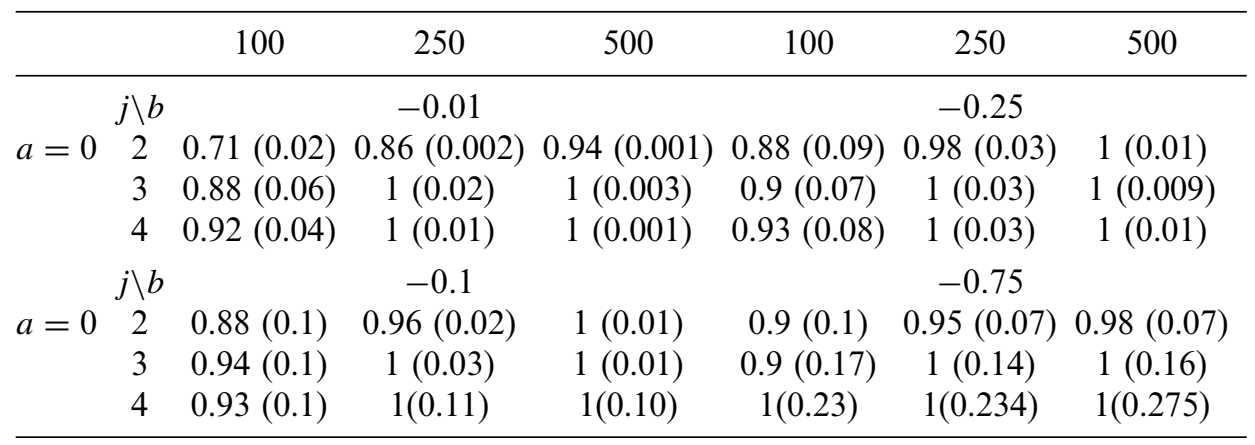

$j=3$ followed by $j=4$ and $j=2$, respectively. The intuition given by Escribano (2004) is the following: cubic polynomials are very flexible and can approximate different level shifts. Furthermore, the error correction term in this case can be equilibrium correcting (stable nonlinear adjustment) and therefore not a deviating error adjustment term as can happen with the quadratic polynomial. Notice that, as expected, the empirical power of the tests decreases with the parameter $a$ even for large negative values of $b$. Let the nonlinear cointegrating function be $g\left(z_{t-1}, \alpha\right)=\exp \left(z_{t-1} / 100\right)$. The size adjusted power of the RCC test and the EG test (in parenthesis) for parameter values $a=0,1$ and $b=$ $-0.01,-0.05,-0.1,-0.25,-0.5,-0.75$, are given in Table 6 .

The power of the EG test is very low since this linear procedure misspecifies the estimation of the cointegrating vector by assuming that it is linear. When there is no short-run dependence $(a=0)$ the power of the RCC is very high and it is reduced when the parameter $a$ is large. This is due to the fact that if the short-run dependence is high, the nonlinearity also strongly affects the short-run dependence of the cointegrating errors and therefore the power of those tests that do not take this information into account is reduced. Similar results are obtained when the

\section{Table 5}

Size adjusted power of the RCC test and EG test (in parenthesis) at 5\% significance level based on $H_{1}$ with $g=z_{t-1}^{j}, j=2,3$, and 4, for different sample sizes $\mathrm{n}$, different values of parameter $b$ and fixing the parameter $a$ at $a=1$

\begin{tabular}{|c|c|c|c|c|c|c|c|}
\hline & & 100 & 250 & 500 & 100 & 250 & 500 \\
\hline & $j \backslash b$ & & -0.01 & & & -0.25 & \\
\hline$a=1$ & 2 & $0.35(0.008)$ & $0.5(0.003)$ & $0.65(0)$ & $0.45(0.057)$ & $0.6(0.02)$ & $0.7(0.005)$ \\
\hline & 3 & & $0.7(0$ & $0.87(0.0$ & $0.6(0$ & 0.8 & $0.9(0$. \\
\hline & 4 & & $0.7(0.01)$ & $0.8(0.002)$ & $0.5(0.09)$ & $0.6(0.02)$ & $0.7(0.005$ \\
\hline & $j \backslash b$ & & -0.1 & & & -0.75 & \\
\hline$a-1$ & 2 & $0.5(0.072)$ & $0.6(0.047)$ & $0.8(0.02)$ & $0.3(0$. & & $0.6(0.057)$ \\
\hline & 3 & & & & & 0.64 & \\
\hline & 4 & $0.55(0.09)$ & $0.7(0.13)$ & $0.8(0.09)$ & $0.3(0.24)$ & $0.45(0.23)$ & $0.6(0.27)$ \\
\hline
\end{tabular}




\section{Table 6}

Size adjusted power of the RCC test and EG test (in parenthesis) at 5\% significance level based on $H_{1}$ with $g=\exp \left(z_{t-1} / 100\right)$ for different sample sizes $n$ and different values of parameters $a$ and $b$

\begin{tabular}{lccccccc}
\hline & \multicolumn{3}{c}{$a=0$} & & \multicolumn{3}{c}{$a=1$} \\
\cline { 2 - 3 } \cline { 7 - 8 }$b \backslash n$ & 100 & 250 & 500 & & 100 & 250 & 500 \\
\hline-0.01 & $0.4(0.06)$ & $0.48(0.05)$ & $0.5(0.05)$ & & $0.4(0.07)$ & $0.45(0.07)$ & $0.46(0.08)$ \\
-0.05 & $0.43(0.05)$ & $0.52(0.05)$ & $0.57(0.05)$ & & $0.43(0.05)$ & $0.46(0.05)$ & $0.5(0.05)$ \\
-0.1 & $0.5(0.05)$ & $0.61(0.05)$ & $0.7(0.05)$ & & $0.43(0.06)$ & $0.5(0.06)$ & $0.6(0.06)$ \\
-0.25 & $0.6(0.05)$ & $0.8(0.06)$ & $0.82(0.09)$ & & $0.5(0.07)$ & $0.54(0.06)$ & $0.7(0.08)$ \\
-0.5 & $0.65(0.07)$ & $0.85(0.09)$ & $0.9(0.1)$ & & $0.52(0.08)$ & $0.57(0.13)$ & $0.76(0.13)$ \\
-0.75 & $0.7(0.1)$ & $0.9(0.11)$ & $1(0.15)$ & & $0.6(0.14)$ & $0.65(0.17)$ & $0.8(0.19)$ \\
\hline
\end{tabular}

nonlinear cointegrating function is $g\left(z_{t-1}, \alpha\right)=\log \left(z_{t-1}+100\right)$; see Garcia (2004). Polynomial transformations, cubic or rational, are flexible functional forms used to study general nonlinear error correction adjustments in asymmetric contexts. Escribano (2004) justified those functional forms based on Pade's approximations. In what follows we analyze the power of the noncointegration tests RCC and EG for nonlinear error correction models.

$H_{1}$ : Linear cointegration with an NEC

DGP:

$$
\begin{gathered}
\Delta y_{t}=c+a \Delta x_{t}+f\left(y_{t-1}-\alpha x_{t-1}\right)+w_{1 t}, \\
\Delta x_{t}=w_{2 t}
\end{gathered}
$$

with $w_{1 t}, w_{2 t} \operatorname{Nid}(0,1)$ errors, mutually independent. Consider the following value of the linear cointegrating parameter $\alpha=1$, with different nonlinear adjustments, $f(\cdot)$. Let the cointegrating error be $U_{t-1}=y_{t-1}-\alpha x_{t-1}$ and let the NEC adjustment be the following polynomial case:

$$
f\left(U_{t-1}\right)=\left(U_{t-1}-0.2\right) U_{t-1}^{2} .
$$

The functional form (17) is justified by the representation theorem of NEC models of Escribano (2004) where the stability condition on the nonlinear adjustment is satisfied $\left(-2<\frac{d f\left(U_{t-1}\right)}{d x_{t-1}}<0\right)$.

It is clear that the power of the RCC is much higher than the power of the EG and that the power of RCC decreases with the short-run dependence of both series measures by parameter $a$. Following Escribano (2004), we combine now both types of linearities.

$H_{1}$ : Nonlinear cointegration in an NEC model

DGP:

$$
\begin{gathered}
\Delta y_{t}=c+a \Delta x_{t}+f\left(y_{t-1}-g\left(x_{t-1}, \alpha\right)\right)+w_{1 t}, \\
\Delta x_{t}=w_{2 t},
\end{gathered}
$$


Table 7

Size adjusted power of the RCC test and the EG

test (in parenthesis) for different values of

parameter $a$ small and $n$ small of model (17)

\begin{tabular}{lccc}
\hline$a \backslash n$ & 100 & 250 & 500 \\
\hline 0 & $0.7(0.04)$ & $0.8(0.03)$ & $0.9(0.02)$ \\
0.5 & $0.6(0.02)$ & $0.7(0.03)$ & $0.8(0.07)$ \\
1 & $0.2(0.08)$ & $0.43(0.02)$ & $0.5(0.01)$ \\
\hline
\end{tabular}

where $w_{1 t}, w_{2 t}$ are $\operatorname{Nid}(0,1)$, mutually independent and where $\alpha=1$. We can consider two types of nonlinearities in the cointegration relationship.

Let $U_{t-1}=y_{t-1}-\log \left(x_{t-1}\right)$ and substitute in (17). The size adjusted power of the RCC and EG tests are given in Table 7 if $U_{t-1}$ is included in (17). Similar power results are obtained for $U_{t-1}=\log \left(y_{t-1}\right)-\alpha x_{t-1}$.

\subsection{Linear Cointegration with Structural Changes in the Cointegrating Vector}

There is a body of literature on the effects of cointegration testing in the presence of structural changes. In what follows we want to simulate different cases based on the DGP of Arranz and Escribano (2001) to evaluate the power of the RCC in this context when the break point is in the middle of the sample. Table 8 shows that the RCC test is clearly more powerful than the EG test in the presence of structural breaks.

$H_{1}$ : Linear error correction and cointegration in the presence of structural change in the cointegrating vector DGP:

$$
\begin{gathered}
\Delta x_{t}=w_{1 t}, \\
\Delta y_{t}=c+a \Delta x_{t}+b\left[y_{t-1}-\left(c_{1} D_{1 t-1} x_{t-1}+\alpha x_{t-1}\right)\right]+w_{2 t},
\end{gathered}
$$

where $w_{1 t}, w_{2 t}$ are $\operatorname{Nid}(0,1)$ errors, and mutually independent, where $c_{1}$ measures the change in the cointegrating vector. We will consider the following values, $\alpha=1, c_{1}=2$. The structural break is created by the artificial dummy variable $D_{1 t}$, defined by

$$
D_{1 t}= \begin{cases}1, & t \geq \frac{n}{2}, \\ 0 & \text { otherwise. }\end{cases}
$$

\section{Table 8}

Size adjusted power of the RCC test and the EG test (in parenthesis) for different values of $a$ and $n$ when $U_{t-1}$ is included in (17)

\begin{tabular}{lccc}
\hline$a \backslash n$ & 100 & 250 & 500 \\
\hline 0 & $0.72(0.02)$ & $0.85(0.032)$ & $0.93(0.04)$ \\
0.5 & $0.6(0.02)$ & $0.7(0.04)$ & $0.8(0.02)$ \\
1 & $0.35(0.09)$ & $0.42(0.021)$ & $0.45(0.012)$ \\
\hline
\end{tabular}


Table 9

Size adjusted power of the RCC test and EG test (in parenthesis) for different values of parameters $a, b, n$ and $c_{1}=2$

\begin{tabular}{|c|c|c|c|c|c|c|}
\hline $\begin{array}{l}c_{1}=2 \\
a \backslash n\end{array}$ & 100 & 250 & 500 & 100 & 250 & 500 \\
\hline & & $b=-0.01$ & & & $b=-0.025$ & \\
\hline & $0.5(0.2)$ & $0.65(0.1)$ & $0.66(0.2)$ & $0.65(0.2)$ & $0.8(0.24)$ & $0.85(0.23)$ \\
\hline 1 & $0.41(0.05)$ & $\begin{array}{l}0.5(0.03) \\
b=-0.5\end{array}$ & $0.64(0.02)$ & $0.54(0.01)$ & $\begin{array}{l}0.61(0.01) \\
b=-0.75\end{array}$ & $0.8(0.007)$ \\
\hline 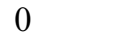 & $0.81(0.23)$ & $1(0.25)$ & $1(0.25)$ & $0.8(0.25)$ & $1(0.26)$ & $1(0.29)$ \\
\hline 1 & $0.62(0.03)$ & $0.8(0.02)$ & $0.9(0.01)$ & $0.61(0.06)$ & $0.73(0.06)$ & $0.91(0.07)$ \\
\hline
\end{tabular}

Based on 10,000 replications of the Monte Carlo simulations, we obtained the size adjusted power of the tests RCC and EG, see Table 9.

Table 9 show that the RCC is more powerful than the EG test to reject the null hypothesis of noncointegration in the presence of a structural break in the cointegrating vector. The power of the RCC is very good for moderate short-run dependence $(a=1)$ and when the parameter $(b)$ of the error correction adjustment is not slow. Those power results of the RCC test statistic are very promising; however, we need to find a small sample correction of the RCC test statistic to make it invariant to the short-run dependence indicated by the parameter $a$. As was mentioned in the previous section, the solution to this problem is in the test statistic that we called $\mathrm{RCC}_{\mathrm{CD}}$ ( $\mathrm{RCC}$ corrected for dependence). The idea of the correction is to count the synchronicity of the jumps, or new records, of both series ( $y$ and $x$ ) but relative to the synchronicity of the jumps in the first differences of the series $(\Delta y$ and $\Delta x$ ). The $\mathrm{RCC}$ with the correction for dependence is $\mathrm{RCC}_{\mathrm{CD}}$ mentioned before. We showed in Table 2 that the critical values of the $\mathrm{RCC}_{\mathrm{CD}}$ test statistic are now independent of the short-run correlation measured by the parameter $a$. The question now is to evaluate the impact of this small sample correction on the power of the test. However, since the empirical distribution of the $\mathrm{RCC}_{\mathrm{CD}}$ is very similar to the RCC with $a=0$, and since for $a=0$ the RCC test was most powerful, we expect also to get important power improvements when $a \neq 0$ by using $\mathrm{RCC}_{\mathrm{CD}}$ instead of RCC.

\section{Empirical Applications}

\subsection{Analysis of Gold and Silver Prices}

Gold and silver have been actively traded for thousands of years and remain important and closely observed markets. Here, following Escribano and Granger (1998), monthly prices are analyzed from the end of 1971 until 1996. We are interested in testing the existence of contemporaneous relationships between the prices of the two commodities in log levels. These prices are determined in clearly speculative markets and therefore their behavior should be captured by unit-root time series models. The unit root is supported by the Dickey Fuller (DF) type tests using from 1 to 6 lags of the dependent variable and including constant and constant and trend variables in the regression equation. There is, however, a feature of this 
data that makes it particularly interesting, which is the widely known and welldocumented bubble in silver prices from roughly June 1979 to March 1980. The objective now is to investigate the effect of the bubble on testing for the existence of a long-run relationship (cointegration). The results in Escribano and Granger (1998) support that the use of the intercept dummies (level shifts in the intercept, introduced to explain the bubble period and their impact on the postbubble period) greatly strengthens the evidence of cointegration.

When testing the null hypothesis using the EG test on the residuals with one lag we get a $t$ ratio $t_{0}=-2.23$ and therefore we cannot reject $H_{0}$ at the $5 \%$ significance since the critical value is -3.7 . If we consider the two level shifts that occur in the cointegration relationship, however, we get $t_{0}=-4.54$, which rejects the null of no cointegration. ${ }^{1}$ Testing for cointegration between the log prices of gold and silver with the RCC test statistics is interesting since those procedures do not require prior estimation of the cointegrating relationship and could indicate departures from linearity.

On the available data sample (of length $n=224$ ), the values obtained for the $\mathrm{RCC}$ and for the $\mathrm{RCC}_{\mathrm{CD}}$ (with $x_{t}, y_{t}$ representing now the logarithms of the gold and the silver price series) were $4.8045(a=0,5 \%$ C.V. $=3.12)$ and $3.56(5 \%$ C.V. $=$ 3.3 ), respectively. These findings support the rejection by $H_{0}$ of independent random walks in favor of the cointegration alternative hypothesis (between the log prices), respectively at the $5 \%$ significance level.

\subsection{Analysis for the UK Money Demand (1878-2000)}

Escribano (1986, 2004) found a cointegration relationship between the log of velocity of circulation of money $(\mathrm{V})$ and short-run interest rates in nominal terms (RNA) from annual observations from 1878 to 2000. Therefore, this cointegration relationship is nonlinear, and he showed that if we transform V and RNA in $\operatorname{logs}$, they are still cointegrated and the cointegration relationship is linear.

However, when we test the null hypothesis of no cointegration between $\log (\mathrm{V})$ and RNA based on the residuals of the EG test statistic we obtain a value of -1.7 and therefore we cannot reject the null hypothesis 5\%. This result contradicts the ECM test done in the nonlinear error correction model. Applying our RCC test statistic, we found $\mathrm{RCC}=3.5$, which is larger than the $5 \%$ critical value, for $n=130$ and $a=0$ (3.11). That is, with the RCC we reject the null of no cointegration if there is no short-run contemporaneous correlation. The same result is obtained for certain values of $a \neq 0$ but not for all. For example, we also reject for $a=0.5$, but not for $a=1$ and 1.5. Therefore, it is important to apply the $\mathrm{RRC}_{\mathrm{CD}}$ test statistic, which is independent of $a$, in order to make a final decision.

\section{Summary}

In this article we proposed a noncointegration test statistic (RCC) based on the first differences of the ranges of the series. This model-free cointegration testing device could also be used in the finite samples. The key idea is to count the synchronicity of the new records, and it is therefore called record counting cointegration (RCC).

${ }^{1}$ There is a body of literature on the effects of structural breaks on unit root and cointegration tests. See, for example, Arranz and Escribano (2001). 
The series will not be cointegrated if there is a lack of synchronicity (up to a constant delay) between the sequences of jumps or between the new records of the series. If the two series have a common stochastic component (common trend), their ranges will tend to jump together, indicating the synchronicity of the new records. The RCC test statistic clearly outperforms the traditional DF unit root test on the cointegrating residuals (EG) as well as nonparametric tests in a similar context previously proposed by Aparicio et al. (2006a). The small sample properties of RCC nonparametric test are analyzed by Monte Carlo simulations and with some empirical examples. In particular, we test the null hypothesis of noncointegration against the alternative hypothesis of a NEC or nonlinear cointegration or both. Furthermore, we are able to show that the RCC test for noncointegration is robust to nonlinearities and structural breaks. One important advantage of this RCC approach is that it does not require previous estimation of the unknown (maybe nonlinear) cointegrating relationship. Our Monte Carlo simulation analysis suggests that the proposed methodology is robust to different departures from the classical linear cointegration context, like nonlinearities in the cointegrating relationship or in the error correction term, or to structrual changes in the cointegrating relationship. The performance evaluation of the RCC in terms of size and power is compared to the EG cointegration test. However, the RCC is sensitive to the short-run dependence between the series. Therefore, we suggest a small sample correction for dependence, the $\mathrm{RCC}_{\mathrm{CD}}$, which works very well even in very small samples (good size and power properties). Finally, our RCC test statistic is applied to different data sets to show its usefulness in identifying cointegration in the presence of important nonlinearities and structural changes.

\section{Appendix}

Lemma 1. Let $x_{t}=\sum_{i=1}^{t} e_{i, 1}$ where $e_{i, 1}$ are continuous i.i.d. random variables with bounded and symmetric pdf, zero means, and finite variances. Suppose that $x_{0}$ also has a bounded $p d f$ and finite variance. And let $J_{(x)}^{(n)}=n^{-1 / 2} \sum_{t=1}^{n} \mathbf{1}\left(\Delta R_{t}^{(x)}>0\right)$. Then we have

$$
\sum_{t=1}^{n} \mathbf{1}\left(\Delta R_{t}^{(x)}>0\right)=O\left(n^{-1 / 2}\right)
$$

Proof. See Aparicio et al. (2006a).

Proof of Theorem 1. Since $x_{t}$ is a random walk we have from Lemma 1,

$$
\begin{aligned}
\sum_{t=1}^{n} \mathbf{1}\left(\Delta R_{t}^{(x)}>0\right)=O\left(n^{-1 / 2}\right) & \Longrightarrow P\left(\Delta R_{t}^{(x)}>0\right)=O\left(t^{-1 / 2}\right) \\
& \Longrightarrow\left[P\left(\Delta R_{t}^{(x)}>0\right)\right]^{2}=O\left(t^{-1}\right) \\
& \Longrightarrow \sum_{t=1}^{n}\left[P\left(\Delta R_{t}^{(x)}>0\right)\right]^{2}=O(\log n)
\end{aligned}
$$

since from Euler's formula we can write $\sum_{t=1}^{n} t^{-1}=\log n+\gamma+\frac{1}{2 n}+\frac{1}{12 n^{2}}+$ $O\left(n^{-4}\right) \gamma=0.57721566$ (Euler's constant).

Now if $x_{t}$ and $y_{t}$ are independent we have 


$$
\begin{aligned}
E\left\{\mathrm{RCC}_{x, y}^{(n)}\right\} & =\sum_{t=1}^{n} P\left(\Delta R_{t}^{(x)}>0\right) P\left(\Delta R_{t}^{(y)}>0\right) \\
& =\sum_{t=1}^{n}\left[P\left(\Delta R_{t}^{(x)}>0\right)\right]^{2}=O(\log n) .
\end{aligned}
$$

Therefore, under $H_{0}$, we can write for some positive constant $\mu$ :

$$
\mathrm{RCC}_{x, y}^{(n)}=\mu \log n+\delta_{n} V
$$

where $V$ denotes a nondegenerate random variable with unit variance and $\delta_{n}$ defines the asymptotic order for the standard deviation of $\mathrm{RCC}_{x, y}^{(n)}$. Our next objective is to determine $\delta_{n}$. To do this, first note that $E\left(\mathrm{RCC}_{x, y}^{(n)}-\mu \log n\right)^{2}=E\left(\delta_{n} V\right)^{2}=\delta_{n}^{2} E(V)^{2}$

$$
\begin{aligned}
E\left\{\left[\mathrm{RCC}_{x, y}^{(n)}\right]^{2}\right\} & =E\left\{\sum_{t=1}^{n} \sum_{t^{\prime}=1}^{n} \mathbf{1}\left(\Delta R_{t}^{(x)}>0\right) \mathbf{1}\left(\Delta R_{t}^{(y)}>0\right) \mathbf{1}\left(\Delta R_{t^{\prime}}^{(x)}>0\right) \mathbf{1}\left(\Delta R_{t^{\prime}}^{(y)}>0\right)\right\} \\
& =\sum_{t=1}^{n}\left[P\left(\Delta R_{t}^{(x)}>0\right)\right]^{2}+2 \sum_{t=1}^{n} \sum_{t^{\prime}=t+1}^{n}\left[P\left(\Delta R_{t}^{(x)} \Delta R_{t^{\prime}}^{(x)}>0\right)\right]^{2} \\
& =\sum_{t=1}^{n}\left[P\left(\Delta R_{t}^{(x)}>0\right)\right]^{2}+W_{x, y}^{(n)},
\end{aligned}
$$

where we let

$$
\begin{aligned}
W_{x, y}^{(n)} & =2 \sum_{t=1}^{n} \sum_{t^{\prime}=t+1}^{n}\left[P\left(\Delta R_{t}^{(x)} \Delta R_{t^{\prime}}^{(x)}>0\right)\right]^{2} \\
& =2 \sum_{t=1}^{n} \sum_{t^{\prime}=t+1}^{n}\left[P\left(\Delta R_{t^{\prime}}^{(x)}>0 \mid \Delta R_{t}^{(x)}>0\right)\right]^{2}\left[P\left(\Delta R_{t}^{(x)}>0\right)\right]^{2} \\
& =2 \sum_{t=1}^{n-1}\left[P\left(\Delta R_{t}^{(x)}>0\right)\right]^{2} \sum_{t^{\prime}=t+1}^{n}\left[P\left(\Delta R_{t^{\prime}-t}^{(x)}>0\right)\right]^{2} .
\end{aligned}
$$

Now we observe that

$$
\begin{aligned}
\sum_{t=1}^{n-1}[ & \left.P\left(\Delta R_{t}^{(x)}>0\right)\right]^{2} \sum_{t^{\prime}=t+1}^{n}\left[P\left(\Delta R_{t^{\prime}-t}^{(x)}>0\right)\right]^{2} \\
& =\mu^{2} \sum_{t=1}^{n-1} t^{-1}(\log n-\log t) \\
& =\mu^{2}(\log n)^{2}-\mu^{2} \sum_{t=1}^{n-1} t^{-1} \log t \\
& =\mu^{2}(\log n)^{2}-\mu^{2}\left\{\sum_{t=1}^{n}\left(\frac{t}{n}\right)^{-1}\left(\log \frac{t}{n}+\log n\right) \frac{1}{n}\right\} \\
& =\mu^{2}(\log n)^{2}-\mu^{2}(\log n)^{2}-\mu^{2}\left\{\sum_{t=1}^{n}\left(\frac{t}{n}\right)^{-1}\left(\log \frac{t}{n}\right) \frac{1}{n}\right\}
\end{aligned}
$$




$$
\begin{aligned}
& \simeq-\mu^{2} \int_{1 / n}^{1} \frac{\log x}{x} d x \\
& =\frac{1}{2} \mu^{2}(\log n)^{2} .
\end{aligned}
$$

It follows:

$$
\begin{aligned}
\operatorname{Var}\left\{\mathrm{RCC}_{x, y}^{(n)}\right\} & \simeq \mu \log n+\mu^{2}(\log n)^{2}-\left[E\left\{\mathrm{RCC}_{x, y}^{(n)}\right\}\right]^{2} \\
& =\mu \log n
\end{aligned}
$$

This entails that

$$
\delta_{n}=O\left((\log n)^{1 / 2}\right) \text { and }(\log n)^{-1} \mathrm{RCC}_{x, y}^{(n)} \rightarrow 1
$$

\section{Acknowledgments}

This research was partially supported by the project SEJ2005-05485, SEJ200506454, and European project HPRN-CT-2002-00232.

\section{References}

Aparicio, F. M. (1995). Nonlinear Modelling and Analysis Under Long-Range Dependence with an Application to Positive Time Series. Ph.D. thesis, Swiss Federal Institute of Technology, Dpt. of Electrical Enginnering, Laussane (Suiza).

Aparicio, F. M., Granger, C. W. J. (1995). Nonlinear Cointegration and Some New Tests for Comovements Modelling (Working Paper 95-15). Dept. of Economics of the University of California, San Diego.

Aparicio, F. M., Escribano, A., Garcia, A. (2004). A Range Unit Root Test. Working Paper of the Department of Statistics of Carlos III University.

Aparicio, F. M., Escribano, A., Garcia, A. (2006a). Range unit root (RUR) tests: Robust against nonlinearities, error distributions, structural breaks and outliers. Journal of Time Series Analysis 27:545-576.

Aparicio, F. M., Escribano, A., Garcia, A. (2006b). Synchronicity Between Financial Time Series: An Exploratory Analysis. Progress in Financial Markets Research. Nova Publishers

Arranz, M. A., Escribano, A. (2001). Cointegration testing under structural breaks: a robust extended error correction model. Oxford Bulletin of Economic and Statistics 62:23-52.

Dickey, A., Fuller, W. (1979). Distribution of the estimators for AR time series with a unit root. Journal of the American Statistical Association 74:427-431.

Engle, R. F., Granger, C. W. J. (1987). Cointegration and error correction: Representation, estimation and testing. Econometrica 55:251-276.

Ericsson, N. R., Hendry, D. F., Prestwich, K. M. (1998). Friedman and Schwartz (1982) revisited: Assesing annual and phase average models of money demand in the United Kingdom. Empirical Economics 23:401-415.

Escribano, A. (1986). Cointegration, Time Co-trends and Error Correction Systems: An Alternative Approach (C.O.R.E. Discussion Paper, 8715). University of Louvain.

Escribano, A. (1987). Error Correction Systems: Nonlinear Adjustments to Linear Long-run Relationships (C.O.R.E. Discussion Paper, 8730). University of Louvain.

Escribano, A. (2004). Nonlinear error correction: the case of money demand in the U.K. (1878-2000). Macroeconomic Dynamics 8:76-116.

Escribano, A., Granger, C. W. J. (1998). Investigating the relationship between gold and silver prices. Journal of Forecasting 17:81-107. 
Escribano, A., Mira, S. (2002). Nonlinear error correction models. Journal of Time Series Analysis 23:509-522.

Franses, P. H., Haldrup, N. (1994). The effects of additive outliers on tests for unit roots and cointegration. Journal of Business and Economic Statistics 12:471-478.

Friedman, M., Schwartz, A. (1982). Monetary Trends in the USA and the UK: Their Relation to Income, Prices, and Interest Rates, 1867-1975. Chicago: University of Chicago Press.

Garcia, A. (2004). Contrastes Basados en Recorridos para Raices Unitarias y Cointegracion. Ph.D. Dissertation. Universidad Carlos III de Madrid.

Granger, C. W. J. (1981). Some properties of time series data and their use in econometric model specification. Journal of Econometrics 16:121-130.

Granger, C. W. J. (1995). Modelling nonlinear relationships between extended memory variables. Econometrica 63(2):265-279.

Granger, C. W. J., Hallman, J. J. (1991). Long-memory series with attractors. Oxford Bulletin of Economics and Statistics 53:11-26.

Hendry, D. F., Ericsson, N. R. (1991). An econometric analysis of UK money demand in monetary trends in the USA and UK by Milton Friedman and Anna J. Schwartz. American Economic Review 81:8-38.

Johansen, S. Estimation and hypothesis testing of cointegration vectors in Gaussian vector autorregresive models. Econometrica 59:1551-1580.

Kremers, J. J., Ericsson, N. R., Dolado, J. J. (1992). The power of cointegration tests. Economics and Statistics 54:325-348.

Leadbetter, M., Rootzén, H. (1988). Extremal theory for stochastic processes. Annals of Probability 16:431-478.

Lindgren, G., Rootzén, H. (1988). Extremes values: theory and technical applications. Scandinavian Journal of Statistics 14:241-279.

Perron, P. (1990). Testing for a unit root in a time series with a changing mean. Journal of Business and Economic Statistics 70:153-162.

Phillips, P. C. B., Perron, P. (1988). Testing for a unit root in time series regression. Biometrika 75:335-346. 